\title{
Lipomas en conducto auditivo interno. Experiencia clínica y revisión del tema
}

\section{Internal auditory canal lipomas. Clinical experience and literature review}

\author{
Freddy Martel V', Caudia Moreno M², Francisca Fernández L', Gabriel Faba C', Constanza Beltrán M¹.
}

\begin{abstract}
RESUMEN
Se presentan dos casos clínicos del Departamento de Aorrinolaringología de la Pontificia Universidad Católica de Chile, con diagnóstico de lipoma del conducto auditivo interno (CAl). Los lipomas del CAl son neoplasias benignas poco frecuentes, con una frecuencia de $0,15 \%$ de los tumores del CAI. Se presentan principalmente entre la tercera y quinta década de la vida, en su mayoría hombres caucásicos. Cínica y audiológicamente son indistinguibles de otros tumores de la misma zona, siendo el neurinoma del acústico (schwanoma vestibular) el tumor más frecuente en el CAI (90\%). Las imágenes de resonancia magnética permiten realizar el diagnóstico específico de lipoma $\theta$ tratamiento quirúrgico ha sido desplazado por el manejo conservador, dado que los lipomas tienen crecimiento lento, sin trasformación maligna, en general, junto con difícil técnica quirúrgica por infiltración a estructuras adyacentes. Sólo estaría indicada la cirugía en casos que exista crecimiento tumoral durante el seguimiento o en pacientes con síntomas intensos y progresivos.
\end{abstract}

Palabras clave: Lipoma, canal auditivo interno, resonancia magnética.

\section{ABSTRACT}

Two case reports with diagnosis of internal auditory canal lipomas are presented. Lipomas of the internal auditory canal are benign neoplasms, making up $0.15 \%$ of all tumors in this area. They occur more frequently in the third to fifth decade of life predominantly in Caucasian males. The are clinically and audiologically indistinguishable from other tumors in this region, with eighth cranial nerve schwannomas (vestibular schwannomas) being the most common IACtumor (90\%). MRI findings allow for specific diagnosis of a lipoma Regarding treatment, surgery has been displaced by a conservative treatment of this rare lesion. Lipomas are slow growth lesions, without malignant transformation, and with inherent difficulties to their removal because of the adipose tissue infiltrating the nerve, as well as only partial resolution of symptoms in most cases. Surgery could only be indicated when relevant and disabling symptoms are present.

Key words: Lipoma, internal auditory canal, magnetic resonance imaging.

1 Médico, Departamento de Otorrinolaringología, Facultad de Medicina, Pontificia Universidad Católica de Chile.

2 Interna de Medicina, Departamento de Oorrinolaringología, Facultad de Medicina, Pontificia Universidad Católica de Chile. 


\section{INTRODUCCIÓN}

Se han descrito diversas lesiones que invaden el conducto auditivo interno (CAI), la gran mayoría de los casos corresponde a neurinoma del acústico $(90 \%)^{2}$, con una incidencia estimada de 1 en 100.000 y en segundo lugar, meningioma $(5-10 \%)^{1}$ sin embargo se han descrito otros tipos histopatológicos, entre los que se encuentran neurinomas del facial, colesteatomas ${ }^{2}$, quiste aracnoideo, papiloma de plexo coroideo, linfoma, angioleiomioma, hemangioma ve noso, angioma cavernoso, glioma pontino, metásta sis, quiste dermoide, epidermoide y lipomas, estos últimos con baja frecuencia de presentación, 0,15\% en $\mathrm{CAl}^{3}$ (30 casos de lipomas en el CAl descritos en la literatura).

\section{MATERIAL Y MÉTODO}

Se presentan dos casos clínicos de lipomas del CAl del Departamento de Otorrinolaringología de la Pontificia Universidad Católica de Chile. Para la revisión del tema se utiliza la base de datos de Pubmed con las palabras claves: lipoma, internal auditory canal, magnetic resonance imaging, resultando una búsqueda de 17 artículos, de los cuales se tiene acceso a 7 de ellos. Con la información de estos artículos se realiza una revisión del tema de lipomas del CAI.

\section{CASOS CLÍNICOS}

\section{Caso 1}

Paciente de 45 años, de sexo masculino, consulta por historia de 3 años de evolución de hipoacusia en oído izquierdo, levemente progresiva, no asociado a vértigo ni tinnitus. Dentro de los antecedentes no existe exposición a ruido. Al examen físico no se encuentran alteraciones de importancia. Se solicita audiometría que muestra hipoacusia sensorioneural de perfil descendente en el oído izquierdo. La resonancia magnética muestra lesión en el CAl del oído izquierdo de 2-3 mm de diámetro, hiperintensa en T1 sin gadolinio e isointensa en T2, compatible con diagnóstico de lipoma del CAl (Figura 1). Con el diagnóstico de lipoma pequeño del CAl, sin sintomatología importante, se decide manejar en forma conservadora. Actualmente se espera que cumpla un año de seguimiento para controlar con una nueva resonancia nuclear magnética.

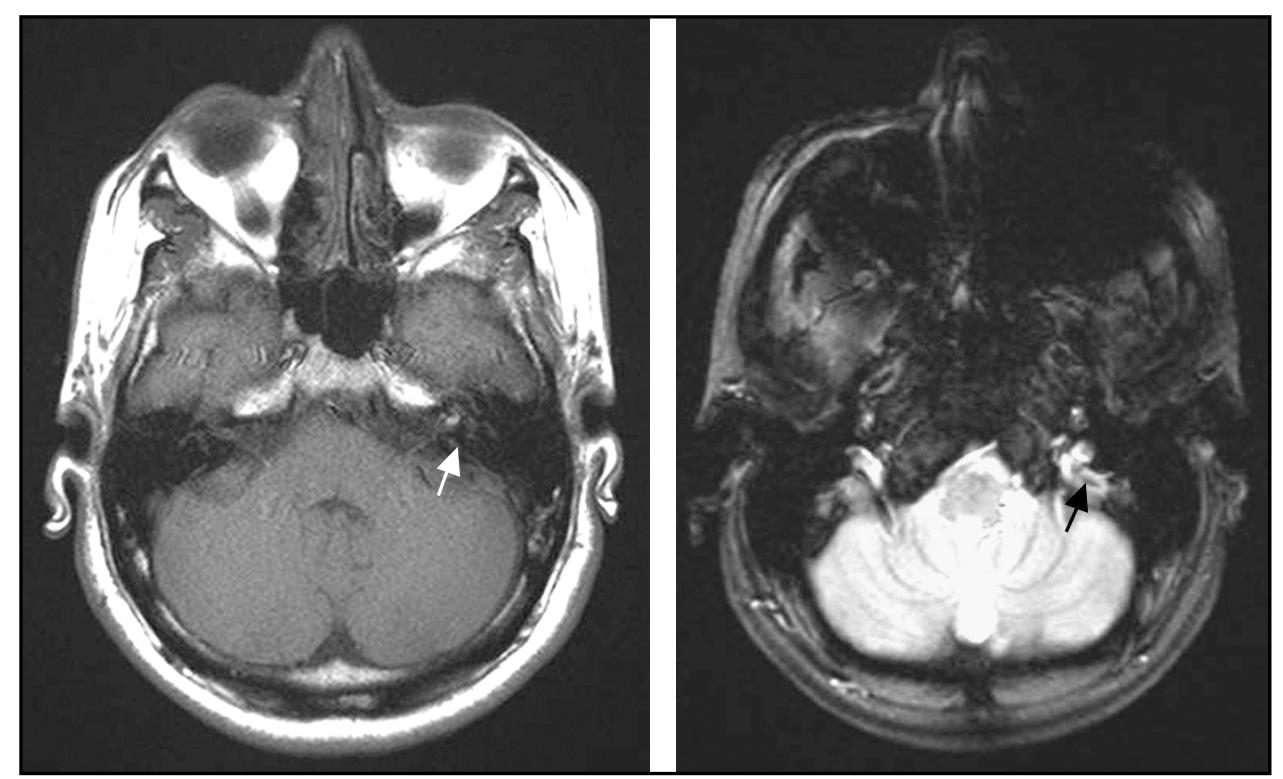

Figura 1. RM, corte axial que muestra T1 a la izquierda, lesión hiperintensa en CAl del oído izquierdo y T2 a la derecha, lesión isointensa del CAl de oído izquierdo. 


\section{Caso 2}

Paciente de 56 años de sexo masculino. Consulta por tinnitus en oído derecho, sin hipoacusia ni vértigo ni antecedentes de exposición a ruido. $\mathrm{日}$ examen físico no presenta alteraciones. Se solicita audiometría que muestra hipoacusia de conducción con gap óseo-aéreo en oído derecho de $20 \mathrm{~dB}$ y timpanograma con curva $\mathrm{Ad}$, por lo que se sospecha una posible disrupción de la cadena osicular. Se solicita TAC de oídos (Fgura 2) para evaluar la cadena osicular, la que se encuentra normal y en forma incidental se pesquisa una lesión hipodensa que deforma el CAl del oído derecho, sugiriendo lipoma del CAl. 1 diagnóstico se confirma con imágenes de resonancia magnética (Figura 3). La audiometría, posresonancia, muestra normalización de la función auditiva Se controla al año con nueva resonancia magnética, la cual muestra una lesión de similares características manteniendo su tamaño de 5 por $9 \mathrm{~mm}$ y sin cambios en su sintomatología, por lo que se mantiene el manejo conservador.

\section{LIPOMAS DEL CONDUCTO AUDITIVO INTERNO}

Los lipomas son neoplasias benignas, que aparecen principalmente entre la tercera y quinta década de la

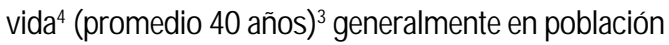
caucásica2, tienen predominio en hombres sobre mujeres en relación 2-5:1, con mayor frecuencia en lado izquierdo $(60 \%)^{5}$. Formados de tejido adiposo maduro, de crecimiento infiltrativo ${ }^{3}$. Tiende a infiltrar fibras nerviosas del VII par craneal y es frecuenteque lo rodeen completamente, extendiéndose en menor medida al VI par craneal'2, siendo difícil disecar los lipomas de estructuras nerviosas adyacentes ${ }^{3}$.

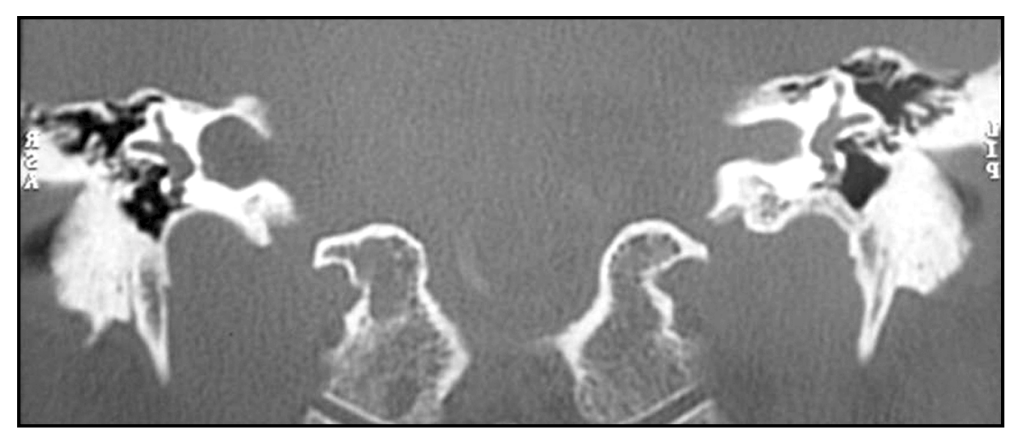

Figura2. TAC de oídos, corte coronal, en el cual se observa imagen que deforma el CAl derecho al comparar con el oído izquierdo.

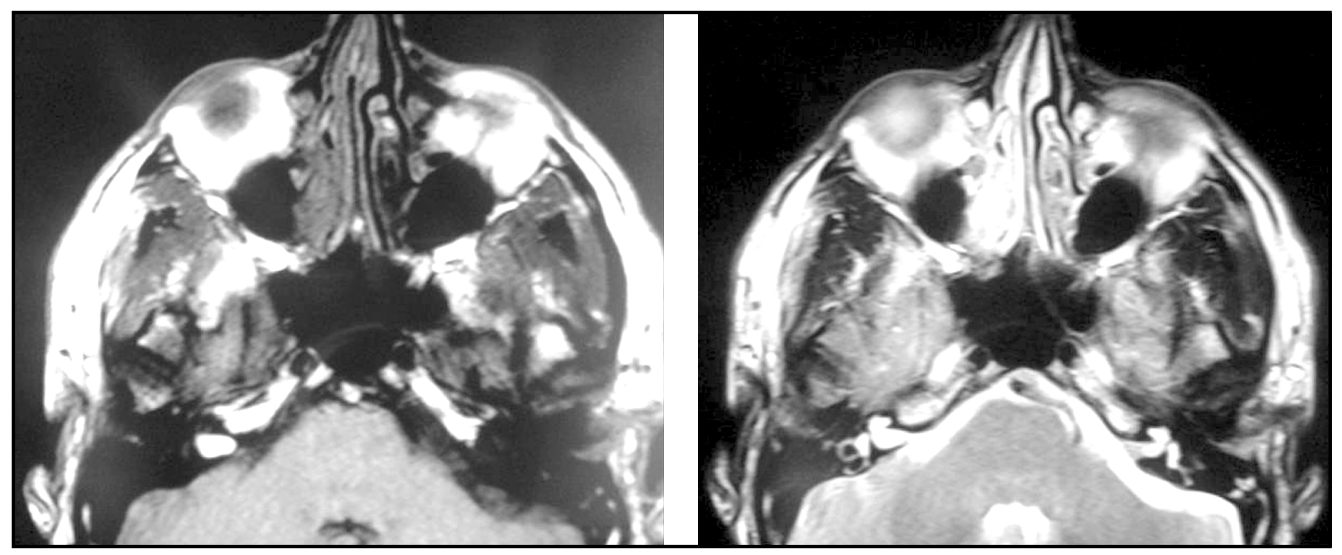

Figura 3. RM, corte axial que muestra T1 a la izquierda, lesión hiperintensa del CAl en oído derecho y T2 a la derecha, lesión isointensa del CAl en oído derecho. 
日 origen de los lipomas en el CAl no se conoce, pero teorías postulan que surgirían por persistencia de un foco anormal de meninge primitiva, que luego se diferencia en tejido adiposo, formando un lipoma. Histológicamente se caracterizan por adipocitos maduros con tejido fibrovascular y fibras nerviosas en su interior 6 .

Cínicamente se presentan síntomas unilaterales: tinnitus, hipoacusiay vértigo ${ }^{7}$. Los síntomas se presentan años antes del diagnóstico, en promedio 4,4 años. Funcionalmente, se presentan con curva audiométrica de hipoacusia sensorioneural descendente, con paresia vestibular ipsilateral. Cínica y audiológicamente son indistinguibles del neurinoma del acústico (schwanoma vestibular) ${ }^{3}$.

Antiguamente, las lesiones del CAl se diagnosticaban en forma retrospectiva, por medio de biopsia posquirúrgica2. Actualmente, en el caso particular de los lipomas del CAl, imágenes de resonancia magnética ayudan a distinguirlos de otros tumores. En la resonancia nuclear magnética los lipomas intracraneales se presentan como le siones de señal hiperintensa y heterogénea en T1, y no se realza con el uso de gadolinio ${ }^{3}$, a diferencia de los neurinomas del acústico que son imágenes hipointensa-isointensa en T1, realzándose fuertemente con el uso de gadolinio. 日 diagnóstico definitivo de lipoma se realiza con técnicas de supresión de grasa en T1, donde la señal hiperintensa cambia a isointensa, siendo la técnica de elección (Tabla 1), esto permite distinguir otras lesiones hiperintensas en T1 como quiste dermoides, melanoma, o hemorragia.

Aras técnicas de diagnóstico como la TC muestran una masa de baja densidad, que sugiere lipoma, pero en este caso, no es específico ${ }^{3}$.

日 tratamiento de los lipomas del CAl antiguamente era quirúrgico, y se realizaba el diagnóstico retrospectivo por medio de anatomía patológica.
Las técnicas quirúrgicas permitían resección completa sólo en $33 \%$ de los casos. Esto permite resolución parcial de los síntomas en $43 \%$ de los pacientes, sin embargo, gran parte de los pacientes (68\%) luego de la cirugía presentaban déficit neurológico severo ${ }^{3}$. La recurrencia es rara si ha existido cirugía ${ }^{3}$.

No existen reportes en la literatura que traten estas lesiones con radiocirugía, a diferencia del schwanoma vestibular.

Considerando que los lipomas del CAl tienen crecimiento lento ${ }^{2}$, sin trasformación maligna en general, junto con difícil técnica quirúrgica por la naturaleza infiltrativa ${ }^{3}$ y con grandes secuelas posquirúrgicas, es posible plantear un manejo conservador con seguimiento periódico de resonancia magnética a los 6-12 meses luego del diagnóstico radiológico, y si persiste del mismo tamaño, en forma anual2 ${ }^{2}$. Sólo en caso que exista crecimiento tumoral importante o en pacientes con síntomas intensos y progresivos estaría indicada la cirugía ${ }^{2,7}$.

En resumen, el diagnóstico correcto de lipoma en el CAl por imágenes (RM), permite importantes cambios en las conductas terapéuticas, desplazando las técnicas quirúrgicas por un tratamiento conservador $^{6}$.

\section{DISCUSIÓN}

Los tumores del conducto auditivo interno son de difícil diagnóstico diferencial, ya que se presentan con sintomatología similar (hipoacusia, tinnitus y vértigo). Lesiones pequeñas se presentan generalmente con examen físico normal, y el estudio funcional de VII par muestra hipoacusia sensorioneural descendente, asociado a paresia vestibular ipsilateral. Fnalmente el diagnóstico definitivo se realiza con resonancia mag-

Tabla 1. Diferencias en imágenes de resonancia magnética entre lipomas del CAl y schwanoma vestibular

\begin{tabular}{|lcc|}
\hline & Lipomas del CAl & Schwanoma vestibular \\
\hline T1 & Hiperintenso & Hipointenso-isointenso \\
T1 Gadolinio & Sin cambios & Se realza \\
T1 Supresión de grasa & Pasa de hiperintenso a isointenso & Sin cambios \\
\hline
\end{tabular}


nética, la cual constituye hoy en día una herramienta eficaz para realizar el diagnóstico diferencial de las lesiones de dicha zona.

En el caso particular de los lipomas del CAl, el diagnóstico utilizando imágenes de resonancia magnética permite un cambio importante en la conducta terapéutica habitual, las técnicas quirúrgicas se reemplazan por tratamiento conservador. La cirugía tiene pobres resultados y deja graves secuela en la función auditiva, además no existe experiencia con otras técnicas quirúrgicas menos invasivas como la radiocirugía, por lo que se hace de vital importancia hacer el diagnóstico de certeza con resonancia magnética para iniciar manejo conservador, que constituye hoy la mejor alternativa de tratamiento.

\section{REFERENCIAS}

1. Darius Kohan, Laura L. Domney, Jin Lim, Noe L, Conev AND ERIC Elowiz. Uncommon lesions presenting as tomors of the internal auditory canal and cerebellopontine angle. The American Journal of Oology 1997, 18: 386-92.

2. Stgan Dazert, Ohristoph Aletser, Dominik Brors,

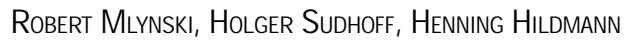
aND JaN Hems. Rare tumors of the internal auditory canal. Eur Arch Atorhinolaryngol (2005) 262: 550-4.

3. Kathlm D, Siechen, MD, Yutaka Sato, MD, PATRICA KIRBY, MD. Internal Auditory Canal Mass in a 46-Year-Od Woman. Arch Pathol Lab Med 2005 Nov; 129(11): 1491-2.

4. Grenwald John H., Lassen Lorenz. Lipomas of the Internal Auditory Canal. The American Laryngological, Rhinological \& Otalogical Society, Inc. Volume 107(3), March 1997; 3648.

5. Bigeow Douglas C., Esen Marc D., Simth Peitr G, Yousem David M., Levine Richard S., Jackler Robert K. Kennedy David W., Kotapka Mark J. Lipomas of the Internal Auditory Canal and Cerebellopontine Angle. The American Laryngological, Rhinological \& Otalogical Society, Inc. October 1998; Volume 108(10), 1459-69.

6. Gan Gaetano Ferri, Gabriela Savastio, and Goavanni Carlo Modugno. Lipoma of the Internal Auditory Canal and Cerebellopontine Angle. Aology \& Neurotology 2004; 25: 1027-8.

7. Krainik A, Onna-Gorse F, Bouocara D, CazalsHateu D, Vilgrain V, Devys A, Rey A, Sterkers O, MeNu Y. MRI of unusual lesions in the internal auditory canal. Neuroradiology 2001 Jan; 43(1): 52-7. 